\title{
Bioconjugates of Microbial Enzyme and Nanoparticles for The Rapid Detection of Analytes
}

\author{
Bi Bi Zainab Mazhari* and Mohammed H Saiemaldahr \\ Department of Microbiology, Saudi Arabia \\ *Corresponding author: Bi Bi Zainab Mazhari, Department of Microbiology, Saudi Arabia
}

\begin{tabular}{|c|c|}
\hline ARTICLE INFO & ABSTRACT \\
\hline Received: 幽 January 30, 2019 & The enormous complexity and diversity of bioconjugates presents a great challenge \\
\hline Published: 㓞February 05, 2019 & $\begin{array}{l}\text { to researchers attempting to reveal their chemical basis and functional aspects. Enzymes } \\
\text { and other biopolymers regulate and perform biochemical functions by binding to ligands. }\end{array}$ \\
\hline $\begin{array}{l}\text { Citation: Bi Bi Zainab Mazhari, Mo- } \\
\text { hammed H Saiemaldahr. Bioconju- } \\
\text { gates of Microbial Enzyme and Na- } \\
\text { noparticles for The Rapid Detection } \\
\text { of Analytes. Biomed J Sci \& Tech Res } \\
\text { 14(1)-2019. BJSTR. MS.ID.002490. }\end{array}$ & $\begin{array}{l}\text { biochemical processes, aiming at the detection of a particular analyte. Qualitative and } \\
\text { quantitative detection of analytes is critical in any target samples for accurate detection. } \\
\text { Analytical biochemistry exploits an intrinsic physicochemical property of the biomolecule } \\
\text { to generate a unique signal, thus circumventing the detection of an analyte. In comparison } \\
\text { to chemical methods biological methods employing bioconjugates are often more } \\
\text { sensitive. Traditional analytical methods require significant biochemical protocols that are } \\
\text { time consuming and require advanced equipments, limiting their applicability. There is an } \\
\text { urgent need to develop stable bioconjugates for economical and rapid detection of analytes } \\
\text { that would be usable in locations far away from laboratories or in a remote geographical } \\
\text { location. Few biosensor applications employ optical techniques such as surface plasmon } \\
\text { resonance to detect binding of analytes to biomolecules immobilized on a surface. }\end{array}$ \\
\hline
\end{tabular}

\section{Introduction}

Bioconjugation is an important field of research resulting into the formulation of bioconjugates for varied applications. Scope of bioconjugation is established in the chemical industry. A prominent example is the use of immobilized nitrile hydratase for the production of acrylamide from acrylonitrile [1]. In biological fields proteins and other biopolymers regulate and perform biological functions by binding to ligands. Discovering and characterizing the natural ligands of biopolymers is crucial to understand biological processes [2]. The bioconjugates of enzyme immobilized on electrode surface has been used for qualitative and quantitative detection of analytes in clinical samples [3]. Reactive functional groups such as primary amines, thiols or carboxylate present on the protein can be coupled to molecules on the nanoparticles surface using any number of cross-linking chemistries [4]. Novel methods for the mild and site-specific derivatization of proteins, DNA, RNA and carbohydrates have been developed for applications such as ligand discovery, disease diagnosis and high through screening $[3,5]$. Biomolecule-nanoparticles conjugates are increasingly important in a wide range of applications including bioanalysis, imaging and nanomedicine.
In particular protein-nanoparticles bioconjugates have been used as electron-dense biospecific stains in electron microscopy for many years [6-10]. Gold nanoparticles were used for extraction of polycyclic aromatic hydrocarbons in drinking water [11]. Liu et al. [12] reviewed the use of nanoparticles for detection and remediation of environmental pollutants. Nanoparticles are frequently employed to aid the detection of environmental pollutants as a preconcentration medium or analytical sensor. These applications take advantage of the unique features of nanoparticles such as their large surface areas and their unique photochemical, electronic or magnetic properties. Chen et al. [13] reviewed the application of nanomaterials and nanotechnology in biosensors. Many novel nanomaterials with unique properties are increasing being exploited to apply for biosensor, improving the property of biosensor and making them higher selectivity and sensitivity, less response time and lower detective limitation. Gold nanoparticles are of great interest due to their fascinating optical properties and their promising applications [14]. Cristina et al. [15] reported different methods for the extraction of phenol constituents from environmental samples which include water, sediments and soil. Gold-coated silica 
nanoparticles were prepared as a preconcentration phase for the determination of mercury in natural water [16]. The presence of gold nanoparticles in biosensor provides a biocompatible microenvironment for biomolecules, greatly amplified the immobilized amount of biomolecules on the electrode surface and improved the sensitivity of the biosensor [17].

\section{Mini Review}

The conjugation of enzymes with gold nanoparticles can lead to the retention or even to an increase of their biological stability/ activity [18]. Enzyme nanoparticles conjugates that take advantage of the catalytic activity of bound enzymes have been reported for bio-analytical and biotechnological applications [19]. Certain phenolic compounds are specific substrates for the enzymes tyrosinase and dehydrogenase and nonspecific for peroxidases and dehydrogenases [20]. Based on the above properties, both electrochemical and optical methods for the detection of pesticides and phenols have been developed. The best performing biosensor in terms of sensitivity, limit of detection, throughout operational and storage stability was reached for one where tyrosinase was immobilized in nafion [20]. The use of gold nanoparticles immobilized together with an enzyme in an electrode membrane have been shown to improve the response of the enzyme electrode where gold nanoparticles of small size allow more freedom in the orientation for the anchored protein molecules and hence maximize the utilization of their bioactive sites [21]. Methods for tyrosinase immobilization reported in the literature include physical adsorption [22], covalent cross linking [23], incorporation within carbon paste [24-25], immobilization in polymer films [26] or composites [27], covalent immobilization on the electrode surface [28-29] and entrapment in a sol gel matrix [30]. Jacqueline and Christine [31] reported the synthesis and characterization of bioconjugates in which the enzyme Malate Dehydrogenase (MDH) and Citrate Synthase (CS) were adsorbed on commercially synthesized gold nanoparticles. Single active bioconjugate was prepared by adsorbing single enzyme on gold nanoparticles where as dual active bioconjugates were prepared in three way by adsorption of MDH followed by CS; by adsorption of CS followed by $\mathrm{MDH}$ and by adsorption of both enzymes from the same solution.

A tyr-AuNps/BDD biosensor was obtained by immobilizing tyrosinase with gold nanoparticles on electro-deposited boron doped diamond (BDD) [32]. Xiangyi etal. [33] prepared bioconjugate of chemically synthesized gold nanoparticles and commercially obtained Horse Raddish Peroxidase and it was characterized by Resonance Light Scattering Correlation Spectroscopy (RLSCS). Development of bioconjugate of tyrosinase gold nanoparticles functionalized with peptide (CALNN) was produced [34] by immobilizing commercially available mushroom tyrosinase with chemically synthesized gold nanoparticles capped with peptide (CALNN). Further, effect of $\mathrm{pH}$ on the activity of tyrosinase and bioconjugate was determined. A novel immobilization strategy based on self-assembled monolayers (SAMS) technique has been used to immobilize tyrosinase for the determination of phenolic compounds. Amperometric tyrosinase biosensor was formed by the spontaneous assembly of thiolor sulphur compounds from solution onto gold electrode. The variables of the experiment such as $\mathrm{pH}$ and applied potential on the amperiometric signal for the enzyme electrode were optimized and also stability of the enzyme electrode was estimate [32]. Kim et al. [35] has prepared tyrosinase gold bionanoconjuagtes on nanostructural gold surfaces. The immobilized bionanoconjugates of tyrosinase and gold nanoparticles prepared by SAM of thiolates on nanostructured gold surfaces gold nanoparticles were functionalized with mercaptoundeconoic acid (MUN) and second conjugated with tyrosinase producing were then adsorbed on the surface of cationic SAM (11-amino-1-Undecanethiol hydrochloride) on the Piezoelectric quartz crystal coated with gold.

\section{Applications of Bioconjugate}

Kinnattura et al. [36] reported the use of nanoparticles for the removal of pesticides as common contaminants in waste water. A novel reaction nanoscale for drinking water purification was done by the use of noble metal nanoparticl. The severely toxic contaminants such as pesticides, halogenated organics, heavy metals and microorganisms, found in drinking water were removed and detected by using nanoparticles. Kinnattura et al. [36] detected the presence of parts per billion (ppb) levels of Chloropyrifos and Malathion, two common pesticides found in the surface water. Enzyme biosensor based on tyrosinase for the detection of phenol compounds have been attracting great interest for fast and simple detection of pesticides $[17,35,37]$ or phenol compounds in food. Duran and Esposito [38] reported that a number of oxidative enzymes from bacteria, fungi and plants to play an important role in numerous waste treatment applications. Tyrosinase which catalyzes the hydroxylation of phenols and dehydrogenation of o-diphenols in an immobilized form exerted an excellent phenol removal. Bevilaqua et al. [39] studied the use of biological and combined biological/ enzymatic treatments in phenol degradation. Biological treatment efficiently degraded effluents up to $99 \%$ in 48 $\mathrm{h}$, whereas enzymatic pretreatment with tyrosinase removes phenol by $25 \%$ after $2 \mathrm{~h}$ of reaction. Caecillia and Henry [40] reported the action of tyrosinase produced from mealworms on phenols.

The action of tyrosinase on catechol formed pink color and after some time gave reddish brown precipitate, this being due to antioxidation to o-benzoquinone which then reacts with the aniline. The buffered solution of phenol, p-cresol, $\mathrm{m}$-cresol and homocatechol by the action of tyrosinase became orange-yellow, orange-brown or reddish brown. A tyrosinase modified solid composite biosensor has been developed and its applications for the determination of phenol and related compounds in environmental samples were recorded [41]. Leona et al. [42] reported the catalytic activity of tyrosinase purified from Psuedomonas putida F6, Streptomyces 
antibioticus and Agaricus bisporus towards mono and diflorinated monophenols. The relationship between monophenol substrate utilized and color intensity of the product was determined by molar extinction co-efficient method [43]. Hamed et al. [44] used purified tyrosinase from Bacillus thurengensis for the decontamination of water polluted with phenol consitutents. Ramiz et al. [45] developed a new type of paper based bioassay for the colorimetric detection of phenolic compounds including phenol, bisphenol A, catechol and cresols present in tap water and river water samples. The sensor is based on layer by layer assembly approach on filter paper by physically trapping the mushroom tyrosinase in these layers. The senor response is quantified as a color change resulting from the specific binding of the enzymatically generated quinine on the paper.

The sensor showed excellent storage stability at room temperature for several months with 92\% residual activity after 260 days of storage. Marcella et al. [46] developed a low cost, portable and disposable paper-based bioassay for phenolic compounds. Mushroom tyrosinase immobilized on filter paper with 3-Methyl-2-benothiazolinone hydrazone (MBTH) detects the phenol in wine by forming stable colored adducts with their enzymatic products. The proposed assay has the advantage of rapidity and simplicity over other detection methods without need of sophisticated instrumentation and trained personnel. Tyrosinase based biosensors might be interesting devices for fast analytical screening of phenols, especially if gold nanoparticles are used as mediators of the direct electron transfer (DET) reaction between enzyme and electrode substrate. Mushroom tyrosinase was used to remove phenol constituents from waste water. The effect of $\mathrm{pH}$ on catalytic activity of tyrosinase was studied. Tyrosinase was unstable under acidic conditions and at elevated temperatures and about 50\% detection was observed at $\mathrm{pH}$ ranging between 5.0 and 8.0 [47]. Kartin et al. [48] has isolated tyrosinase from Streptomyces antibioticus for the preparation of bi enzymatic biosensor with glucose dehydrogenase for phenol detection. Sung et al. [49] developed a highly sensitive mushroom tyrosinase based amperometric biosensor using biologically designed gold nanowires (AuNws) for pesticides detection. Narayan and Agarwal [50] reviewed the current research based on removal of phenol from waste streams based on class oxidoreductases such as peroxidase, polyphenoloxidase (tyrosinase) and laccasses. Svitel and Miertus [51] developed tyrosinase based biosensor following electrochemical method for the determination of phenol and related compounds in environmental samples. A biosensor was described for the detection of phenolic compounds in water and it was constructed by immobilizing tyrosinase in a thin layer of Eastman AQ polymer on the surface of a screen-printed electrode [52]. Tyrosinase based biosensor was developed for the detection of phenol constituents by immobilizing tyrosinase on colloidal gold modified carbon paste electrode [53].
The stability of biosensor is usually considered as one of the key factor considerably hampering the practical applicability of biosensor. The operation stability of biosensor was tested by repetitive measurements during one month [41]. Duran et al. [38] reported that amino acids bind effectively to gold nanoparticles through the amine groups have been used in the immobilization of proteins and enzymes on gold nanoparticles, as means of developing a simple biocatalyst with good reuse characteristics, $\mathrm{pH}$, temperature and stability. Immobilization of enzymes directly on gold nanoparticles in solution yielded excellent catalytic activity of the enzymes and in many cases enhancement in the enzyme thermal stability as well. A more practical and still highly sensitive detection method based on nanostructured surfaces demonstrates immense promise [31]. In this direction, understanding the surface chemistry of the biogenic nanoparticles is most important in the development of a desired bioconjugate for the detection of phenol constituents from the polluted environmental samples. Enzymenanoparticles conjugates are increasingly important in a wide range of applications mainly bioanalysis.

\section{References}

1. Kobayashi M, Nagasawa T, Yamada H (1992) Enzymatic synthesis of acrylamide: a success story not yet over. Trends Biotechnol 10: 402-408.

2. Rup B, O'Hara D (2007) Critical ligand binding reagent preparation/ selection: When specificity depends on reagents. AAPS J 9(2): 148-155.

3. Jeet Kalia, Ronald T, Raines (2010) Advances in Bioconjugation. Current Organic Chemistry. 14(2): 138-147.

4. Hermanson GT (1996) Bioconjugate Techniques Academic Press: San Diego CA.

5. Rusmini F, Zhong Z, Feigen J (2007) Protein immobilization strategies for protein biochips. Biomacromolecules 8(6): 1775-1789.

6. Willner I, Baron R, Willner B (2007) Integrated nanoparticles biomolecule systems for biosensing and bioelectronics. Biosens. Bioelectron 22: 1841-1852.

7. Hayat MA (1989) Colloidal Gold Principles, Methods and Applications. Academic Pres Inc San Diego CA: 1-3.

8. Katz E, Willner I (2004) Integrated nanoparticle biomolecule hybrid systems: synthesis, properties and applications. Angew Chem Int Ed 43(45): 6042-6108

9. Wang H, Campiglia AD (2008) Determination of polycyclic aromatic hydrocarbons in drinking water samples by soild phase nanoextraction and high-performance liquid chromatography. Anal Chem 80(21): 82028209.

10. Liu H, Crooks RM (2011) Three-dimensional paper microfluidic devices assembled using the principles of origami. J Am Chem Soc 133(44): 17564-17566

11. Chen S, Huang J, Du D, Li J, Tu H, et al. (2011) Methyl Parathion Hydrolase Based Nanocomposite Biosensors for Highly Sensitive and Selective Determination of Methyl Parathion. Biosensors and Bioelectronics 26(11): 4320-4325

12. Xia M, Chen C, Long M, Chen C, Cai W, Zhou B, et al. (2011) Magnetically separable mesoporous silica nanocomposite and its application in Fenton catalysis Micropor. Mesopor Mater 145: 217-223.

13. Chen S, Huang J, Du D, Li J, Tu H, (2011) Methyl Parathion Hydrolase Based Nanocomposite Biosensors for Highly Sensitive and Selective 
Determination of Methyl Parathion. Biosensors and Bioelectronics 26(11): 4320-4325.

14. Xia M, Chen C, Long M, Chen C, Cai W, Zhou B, et al. (2011) Magnetically separable mesoporous silica nanocomposite and its application in Fenton catalysis Micropor. Mesopor Mater 145: 217-223.

15. Cristina Mahugo Santana, Zoraida Sosa Ferrera, Esther Torres M, Torres Padron, Jose Juan Santana Rodriguez, et al. (2009) Methodologies for the extraction of phenolic compounds from environmental samples. New approaches J Molecules 14(1): 298-320.

16. Leopold K, Foulkes M, Worsfold PJ (2009) Gold-coated silica as a preconcentration phase for the determination of total dissolved mercury in natural waters using atomic fluorescence spectrometry. Anal Chem 81(9): 3421-3428.

17. Kim GY, Kang MS, Shim J, Moon SH (2008) a Substrate bound tyrosinase electrode using gold nanoparticles to pyrroloquinoline quinone for a pesticide biosensor. Sens Actuators B Chem 133(1): 1-4.

18. You CC, Verma A, Rotello VM, (2006) Engineering the nanoparticles -biomacromolecule interface. Soft Matter 2: 190-204.

19. Kreft O, Prevot M, Mohwald H, Sukhorukov G (2007) Shell-in-shell microcapsules: A novel tool for integrated, spatially confined enzymatic reactions. Angw Chem Int Ed 46(29): 5605-5608.

20. Nistor C, Emneus J, Gorton L, Ciucu (1999) A improved stability and altered selectivity of tyrosinase based graphite electrodes for detection of phenolic compounds. Anal Chem Acta 387(3): 309-326.

21. Chen Gui, Xuan Dai, Daxiang Cui, (2006) Advances of Nanotechnology Applied to Biosensors. Nano Biomed. Eng pp. 2150-5578.

22. Hall GF, Best DJ, Turner APF (1988) The determination of p-cresol in chloroform with an enzyme electrode used in the organic phase. Anal Chim Acta 213: 113-119.

23. Eggins BR (2002) Chemical Sensors and Biosensors. Chichester John Wiley and Sons.

24. Wang J, Fang D, Lopez D, (1994) Ampherometric biosensor for phenols based on tyrosinase graphite-epoxy biocomposite. Analyst 199(3): 455458.

25. Liu J, Hansen C, Quake SR, (2003) Solving the world-to-chip interface problem with a microfluidic matrix. Anal Chem 75(18): 4718-4723.

26. Apetrei P, Alessio CJL, Constantino JA, De Saja, Rodriguez-Mendez ML, et al. (2011) Biomimetic biosensor based on lipidic layers containing tyrosinase and lutetium bisphthalocyanine for the detection of antioxidants. Biosens Bioelectron 26(5): 2513-2519.

27. Lu W, Huang Q, Ku G, Wen X, Zhou M (2010) Photoacoustic imaging of living mouse brain vasculature using hollow gold nanospheres. Biomaterials 31(9): 2617-2626.

28. Zhao H, Huang J (2006) Multilayer membranes for glucose biosensing via layer-by-layer assembly of multiwall carbon nanotubes and glucose oxidase. Anal Biochem 350(1): 138-144.

29. Zhao W, Chiuman W, Lam JC, Brook MA, Li Y, et al. (2007) Simple and rapid colorimetric enzyme sensing assays using non-cross-linking gold nanoparticle aggregation. Chem Commun Pp. 3729-3731.

30. Kochana J, Gala A, Parczewski A, Adamski J, (2008) Titania sol-gelderived tyrosinase-based amperometric biosensor for determination of phenolic compounds in water samples, Examination of interference effects. Analytical and Bioanalytical Chemistry 391(4): 1275-1281.

31. Jacqueline D, Keighron , Christine D, Keating, (2010) Enzyme: Nanoparticle Bioconjugates with Two Sequential Enzymes: Stoichiometry and Activity of Malate Dehydrogenase and Citrate Synthase on Au Nanoparticles. Langmuir 26(24): 18992-19000.

32. Bruno C Janegitz, Roberta A, Medeiros, Romeu C, Rocha-Filho, et al (2012) Direct electrochemistry of tyrosinase and biosensing for phenol based on gold nanoparticles electrodeposited on a boron-doped diamond electrode. Diamond and Related Materials 25: 128-133.
33. Xiangyi Huang, Tao Lan, Bocheng Zhang, Jicun Ren, et al. (2012) Gold nanoparticle-enzyme conjugates based FRET for highly sensitive determination of hydrogen peroxide, glucose and uric acid using tyramide reaction. Analyst 137(16): 3659-3666.

34. Cortez J, Vorobieva E, Gralheira D, Osorio I, Soares L, et al. (2011) Bionanoconjugates of tyrosinase and peptide-derivatised gold nanoparticles for biosensing of phenolic compounds. J Nanopart Res 13(3): 1101-1113.

35. Kim GY, Shim J, Kang MS, Moon SH, (2008) Preparation of a highly sensitive enzyme electrode using gold nanoparticles for measurement of pesticides at the ppt level. J Environ Monitor 10(5): 632-637.

36. Kinnatura P Lisha, Anshup, Pradeep T (2009) Enhanced visual detection of pesticides using gold nanoparticles. Journal of Env Sci Health Pesticides Food Contaminants and Agricultural Wastes 44(7): 697-705.

37. Vidal JC, Esteban S, Gil J, Castillo JR (2006) A comparative study of immobilization methods of a tyrosinase enzyme on electrodes and their application to the detection of dichlorvos organophosphorus insecticide. Talanta 68(3): 791-799.

38. Duran N, Marcato PD, Alves OL, Desouza GIH, Esposito E, et al. (2005) Mechanistic aspects of biosynthesis of silver nanoparticles by several Fusarium oxysporum strains. Journal of Nanobiotechnology 3: 1-7.

39. Bevilaqua JV, Cammarota MC, Freire DMG, Santanna JR (2002) Phenol removal through combined biological and enzymatic treatments. Braz J Chem Eng 19: 151-158.

40. Caecillia Elisabeth, Mary Pugh, Henry Stanley Raper (1927) The action of tyrosinase on phenols. CLXXX Thesis University of Manchester 21(6): 1370-1383.

41. Svitel J, Miertus S (1998) Development of tyrosinase-based biosensor and its application for monitoring of bioremediation of phenol and phenolic compounds. Environ Sci Technol 32(6) 828-832.

42. Leona B Martin, Jasmina Nikodinovic, Aoife M McMahon, Erik Vijgenboom, Kevin EO Connor, et al. (2008) Assessing the catalytic activity of three different sources of tyrosinase: A study of the oxidation of mono- and diflourinated monophenols. Enzyme and Microbial Technology 43: 297-301.

43. Fling M, Horowitz NH, Heinemann SF (1963) The isolation and properties of crystalline tyrosinase from Neurospora. J Biol Chem 238: 2045-2053.

44. Hamed M El-Shora, Metwally (2008) Use of tyrosinase enzyme from Bacillus thuringiensis for the decontamination of water polluted with phenols. J Biotechnology 7(2): 305-310.

45. Ramiz SJ, Alkasir, Maryna Ornatska, Silvana Andreescu, et al. (2012) Colorimetric Paper Bioassay for the Detection of Phenolic Compounds. Anal Chem 84(22): 9729-9737.

46. Marcella Arciuli, Gerardo Palazzo, AnnaGallone, Antonia Mallardi (2013) Bioactive paper platform for colorimetric phenols detection. Sensors and Actuators B Chemical 186: 557-562.

47. Keisuke Ikehata, James A Nicell (2009) Color and toxicity removal following Tyrosinase-Catalyzed Oxidation of Phenols. Biotechnology Progress.

48. Katrin Streffer, Erik Vijgenboom, Armand WJW, Tepper, Alexander Makower, et al. (2001) Determination of phenolic compounds using recombinant tyrosinase from Streptomyces antibioticus. Analytica Chimica Acta 427(2): 201-210.

49. Sung-Hee Shin, Gha-Young Kim, Joonmok Shim, Jungok Kim, Hor-Gil Hur, et al. (2012) Use of biologically designed gold nanowire for biosensor application. Korean Journal of Chemical Engineering 29(12): 1666-1669.

50. Narayan AV, Agrawal Pushpa (2012) Enzyme based processes for removal of phenol from waste water: Current status and future challenges. J Environ Res Develop 7:2.

51. Schmidt S (1998) Biodegradation of diaryl ether pesticides, from: Biodegradation of dioxins and furans. chapter 8: 229-281. 
52. Liu J, Mattiasson B (2002) Microbial BOD Sensors for Wastewater Analysis. Water Research 36(15): 3786-3802.

\section{ISSN: 2574-1241}

DOI: 10.26717.BJSTR.2019.14.002490

Bi Bi Zainab Mazhari.Biomed J Sci \& Tech Res

(C) This work is licensed under Creative

Submission Link: https://biomedres.us/submit-manuscript.php
53. Gayathri B Seetharam, Bradley A Saville (2003) Degradation of phenol using tyrosinase immobilized on siliceous supports. Water Research 37(2): 436-440.

\begin{tabular}{ll} 
BIOMEDICAL & Assets of Publishing with us \\
RESEARCHES & - Global archiving of articles \\
& - Immediate, unrestricted online access \\
\hline ISSN: 2574-1241 & - Rigorous Peer Review Process \\
\end{tabular}

